\title{
LA INICIATIVA OPEN ACCESS, UNA VISIÓN DE CONJUNTO
}

\author{
JAVIER SANZ-VALERO*, MARCELO JOSÉ D’AGOSTINO**, \\ LUIS DAVID CASTIEL***, JORGE VEIGA DE CABO****
}

(*) Departamento de Enfermería Comunitária, Medicina Preventiva y Salud Pública e Historia de la Ciencia, Universidad de Alicante. Alicante. España.

(**) Área de Información y Gestión del Conocimiento. Organización Panamericana de la Salud, Oficina Regional de la Organización Mundial de la Salud (OPS/OMS). Washington DC. EEUU.

(***) Departamento de Epidemiología y Métodos Cuantitativos en Salud. Escuela Nacional de Salud Pública. Fundación Oswaldo Cruz (FIOCRUZ). Rio de Janeiro (RJ). Brasil.

(****) Escuela Nacional de Medicina del Trabajo. Instituto de Salud Carlos III. Madrid. España.

\begin{abstract}
RESUMEN
Desde tiempos remotos hasta la Declaración de los Objetivos de Desarrollo del Milenio el tema de Acceso a Información ha sido fundamental para el desarrollo económico y Social en el Mundo y debe ser tratado como un derecho inherente de las personas. El movimiento de Open Access contribuye a otorgar a la comunidad científica la hegemonía que le corresponde, al facilitar el uso de la literatura científica relevante y dar a los autores y a sus trabajos una nueva visibilidad mensurable, eliminando barreras y disminuyendo las inequidades en el acceso a información técnico-científica a través de Internet.
\end{abstract}

\section{PALABRAS CLAVES}

Acceso a información, Open Access, Derechos humanos, literatura científica, Investigación, publicaciones científicas.

\section{ABSTRACT}

From ancient times to the Millenium Development Goals Declaration, the issue of Access to Information has been essential to the world economic and social development and should be tackled as a right inherent to human beings. The Open Access movement contributes to giving the scientific community the hegemony they deserve by facilitating the use of relevant scientific literature and giving the authors and their work a new measurable visibility, eliminating barriers and reducing inequities in the access to technical-scientific information via the Internet.

\section{KEY WORDS}

Information access, Open Access, Human Right, scientific literature, research, scientific publishing. 


\section{ACCESO A INFORMACIÓN COMO UN DERECHO INHERENTE DE LAS PERSONAS}

La sociedad de la información plantea nuevas formas de vida, de trabajo y de interrelaciones entre personas, empresas, gobiernos y organizaciones. También plantea como uno de sus desafíos poder encauzar el potencial de las tecnologías de información y comunicaciones para promover los «Objetivos de Desarrollo de la Declaración del Milenio». A todos los actores involucrados recomienda facilitar el acceso a los conocimientos médicos mundiales y al contenido de carácter local para fortalecer la investigación en materia de salud y programas de prevención públicos que permitan promover la salud de las mujeres y los hombres en todo el continente.

Cuando vemos que cada año mueren 11 millones de niños menores de 11 años, la mayoría de ellos en países en desarrollo, de los que las $2 / 3$ de estas muertes podrían prevenirse con intervenciones ya conocidas $^{1}$, afirmamos que el acceso a Información en Salud debe ser visto y tratado como un Bien Público Regional y un Derecho Inherente de las Personas y aquí el movimiento de Open Access juega un rol fundamental.

En la actualidad, son ya muchos los países del mundo que cuentan con legislación que otorga a la ciudadanía el «Derecho Legal del Acceso a Información Pública» bajo los principios de igualdad, publicidad, celeridad, informalidad y gratuidad, sin embargo, aun queda mucho camino por recorrer para que el acceso a información, principalmente en salud, sea parte integral de los derechos humanos.

\section{¿QUÉ ES LA INICIATIVA «OPEN ACCESS»?}

El término Open Access se utiliza para definir plataformas de acceso a fuentes de información científica como pueden ser monografías, revistas, tesis, manuales de práctica clínica y cualquier otra modalidad de publicaciones editadas en formato electrónico. El acceso se realiza a través de Internet, permitiendo realizar búsquedas, lectura, recuperación de documentos, copia, impresión, distribución y enlace a accesos directos de textos completos de las fuentes, mediante un sistema de acceso libre, directo, permanente y gratuito.

Este movimiento, The Open Access Iniciative $(O A I)$, supone además un compromiso con la calidad de contenidos, por lo que es compatible con la revisión por pares, la impresión, la preservación de contenidos y otras características asociadas a la literatura académica convencional. La diferencia principal se encuentra en que el lector, que en la mayoría de las ocasiones coincide con el productor de conocimiento científico, no se encuentra obligado a pagar por el acceso a la información, disminuyendo así las barreras para la investigación frente a las restricciones de acceso y difusión de sus resultados impuestas por los sistemas más convencionales de los grupos editoriales ${ }^{2}$.

Otra diferencia importante entre el sistema tradicional de publicación utilizado por las grandes editoriales y las plataformas Open Access, se refiere a las condiciones que cada uno de ellos imponen a los autores en lo que se refiere a la "propiedad intelectual». En la mayor parte de las ocasiones, los autores se ven obligados a ceder a las editoriales los derechos de autor del manuscrito que envían para su publicación. Por el contrario, el OAI tiene por norma el respeto ante la propiedad intelectual y el mantenimiento del copyright por el autor.

Por tanto, podríamos resumir que el OAI se encuentra comprometido con la calidad de los contenidos, con la garantía de accesibilidad a la información científica, con el mantenimiento de archivos que preserven el conocimiento, con la eliminación de la obligatoriedad de cesión del copyright de los artículos publicados, y por último, con todos los principios éticos relacionados con la investigación y la publicación científica de documentos ${ }^{3}$.

\section{¿CÓMO, CUANDO Y POR QUÉ SURGE LA INICIATIVA OPEN ACCESS?}

El OAI tiene su origen lejano en iniciativas tendentes a almacenar información de calidad de distintas colecciones y fuentes de conocimiento del Open Society Institute $(\mathrm{OIS})^{4}$, fundación creada en 1993 por el investigador y filántropo George Soros, al objeto de promover sociedades, que al amparo de políticas gubernamentales, permitieran dar soporte a proyectos relacionados con la educación, multimedia, salud pública, derechos de la mujer, reformas legales, sociales y económicas.

Sin embargo, el verdadero lanzamiento del OAI como tal, surge a partir de la reunión que mantuvieron el 1 y 2 de diciembre de 2001, en Budapest, los representantes científicos de diferentes organizaciones, instituciones, asociaciones internacionales y representantes de diferentes países del mundo. Esta reunión, continuidad e otras anteriores, se marcaba como principal objetivo unir esfuerzos y aglutinar iniciativas separadas dentro de un plan estratégico que permitiese generar archivos exhaustivos de fuentes de conocimiento y de los logros recientes 
alcanzados, estableciendo como pieza central la utilización las fuentes de información de OIS como herramienta fundamental del $\mathrm{OAI}^{5}$. Esta iniciativa aporta a la comunidad investigadora un nuevo poder, al facilitar el uso la literatura científica relevante, y que da a los autores y a sus trabajos una nueva visibilidad mesurable, eliminando las barreras, especialmente las del precio.

Así, el 14 de febrero de 2002, nace Budapest Open Access Initiative (BOAI) como una declaración de principios, estrategias y compromisos que fue suscrito por 270 organizaciones y 3.400 particulares en apoyo de la iniciativa. En junio de 2004, el OIS destina una partida presupuestaria importante al soporte del proyecto Open Access, desarrollándose así herramientas como el Directory of Open Access Journals (DOAJ), software, guías, una gerencia para la captación de recursos y de financiación, dotaciones presupuestarias destinadas a organización de conferencias, seminarios, talleres, así como una partida importante para publicaciones ${ }^{6}$.

El éxito de la OAI, se debe en parte al soporte de OIS y a la infraestructura con la que fue dotada a partir de BOAI, pero sobre todo, por la enorme acogida con la que fue recibida dentro de la comunidad científica internacional.

\section{¿CÓMO FUNCIONAN Y QUÉ MODALIDADES PODEMOS ENCONTRAR DENTRO DE LAS PLATAFORMAS OPEN ACCESS?}

El elemento fundamental de Budapest Open Access Initiative (BOAI), lo constituye los e-prints, publicaciones científicas en formato digital, los cuales pueden no haber pasado una revisión por pares (preprints), o haberla pasado (postprints), con lo que se encontraría listo para su publicación y difusión ${ }^{7}$.

Los e-prints, convenientemente almacenados pueden adoptar dos modalidades de presentación:

1. Autoarchivo (self-archiving), en el que los propios autores depositan sus trabajos en repositorios, que pueden ser temáticos o institucionales. Los temáticos, se corresponderían con el concepto de Bibliotecas Virtuales y los mejores ejemplos los encontramos en Eprints in Library and Information Science (E-LIS) y el Localizador de Información de Salud (LIS), de la Biblioteca Virtual de Salud (BVS). Los institucionales, suelen ser una modalidad muy extendida entre Universidades, Organismos e Instituciones públicas
2. Repositorios de revistas con acceso abierto (open access), dentro de los cuales podemos distinguir tres modalidades:

2.1. Plataformas de acceso a revistas editadas exclusivamente en formato electrónico, entre las que podemos citar como más importantes BioMed Central y PLoS Medicine.

2.2. Plataformas de revistas editadas en formato papel y electrónico, entre las que podemos destacar SciELO y DOAJ.

2.3. Plataformas que permiten el acceso electrónico después de un tiempo (llamado tiempo de carencia o de embargo) a la versión de la revistas editada previamente en papel. En este grupo encontramos como más significativas, PubMed Central y otras desarrolladas por diferentes empresas editoriales que utilizan este sistema, como New England Journal of Medicine o Lancet ${ }^{8}$.

\section{PRINCIPALES PLATAFORMAS OPEN ACCESS}

\author{
Access to Global Online Research in Agricul- \\ ture (AGORA) \\ http://www.aginternetwork.org/es/
}

Organización de las Naciones Unidas para la Agricultura y la Alimentación (FAO) y apoyo de sectores privados. Permite acceso a colecciones bibliográficas digitales en los campos de la alimentación, agricultura, ciencia medioambiental y ciencias sociales.

\section{Bioline International}

http://www.bioline.org.br/

Centro de Referência em Informação Ambiental (CRIA), Open Society Institute y la Universidad de Toronto. Permite el acceso libre a publicaciones científicas con revisión por pares de países en vía de desarrollo.

\section{BioMed Central \\ http://www.biomedcentral.com/}

Iniciativa independiente del Reino Unido, proporciona acceso a resultados de investigación biomédica y a unas 150 revistas científicas de calidad y con sistema de revisión por pares. 


\section{Directory of Open Access Journals (DOAJ) \\ http://www.doaj.org/}

Lund University Libraries (Suecia). Directorio de revistas de libre acceso a texto íntegro de publicaciones de calidad contrastada.

\section{Eprints in Library and Information Science (E-LIS) \\ http://eprints.rclis.org/}

Ministerio Español de Cultura en colaboración con el Consorzio Interuniversitario de Lombarda per Elaborazione Automatica (CILEA). Archivo temático de documentos especializados en áreas de bibliotecología y ciencias de la información.

\section{Health InterNetwork Access to Research Initiative (HINARI) \\ http://www.who.int/hinari/es/}

Organización Mundial de la Salud (OMS) y en colaboración con grupos editoriales. Permite acceso a una amplia colección de publicaciones del ámbito biomédico de más de 100 países en vías de desarrollo.

\section{Latindex}

http://www.latindex.org/

Red cooperativa de instituciones de la Región (Coordinada en México). Base de datos bibliográfica con acceso a texto completo de revistas científicas de Latinoamérica, el Caribe, España y Portugal.

\section{Los Alamos Preprint Archive (arXiv) \\ http://arxiv.org/}

Universidad de Cornell (Nueva York) y la National Science Foundation. Plataforma de acceso libre de publicaciones especializadas en física, matemáticas, informática, y biología cuantitativa.

\section{Online Access to Research in the Environment (OARE)}

http://www.oaresciences.org/es/

Iniciativa mixta público-privada entre el Programa de las Naciones Unidas para el Medio Ambiente (PNUMA), la Universidad de Yale y grupos editoriales. Permite el acceso a los países en desarrollo de manera gratuita a una de las colecciones más extensas de literatura sobre ciencias de la tierra y del medio ambiente.

\section{Open Access Repositories (OpenDOAR) \\ http://www.opendoar.org/index.html}

Universidad de Nottingham.. Directorio por autoridades de repositorios académicos en sistema de libre acceso.

\section{Public Library of Science (PloS) \\ http://www.plos.org/}

Organización sin ánimo de lucro integrada por investigadores y científicos de diferentes países comprometidos con el sistema open access, en cooperación con las diversas fundaciones, universidades, Open Society Institut (OSI) y Joint Information Systems Committee (JISC). Al igual que BioMed Central, utiliza un sistema de suscripción por instituciones o soporte directo de la publicación..

\section{PubMed Central \\ http://www.pubmedcentral.nih.gov/}

Centro Nacional para la Información de la Biotecnología (NCBI) de la National Library of Medecine (NLM) de Bethesda (EEUU). Permite acceso libre a publicaciones científicas de más de 200 revistas.

\section{Scientific Electronic Library Online (SciELO) http://www.scielo.org/index.php?lang=es}

Centro Latinoamericano y del Caribe de Información en Ciencias de la Salud, de la Organización Panamericana de la Salud - Organización Mundial de la Salud (BIREME/OPS/OMS). Su objetivo es contribuir al la difusión y el acceso de las publicaciones de calidad a la comunidad científica internacional.

\section{SciELO España \\ http://scielo.isciii.es/scielo.php/lng_es}

Biblioteca Nacional de Ciencias de la Salud del Instituto de Salud Carlos III de Madrid. Facilita acceso a una colección de revistas científicas españolas de ciencias de la salud, seleccionadas de acuerdo a unos criterios de calidad preestablecidos.

\section{The Scholarly Publishing and Academic Resources Coalition (SPARC) \\ http://www.arl.org/sparc/}

Asociación de las Bibliotecas de la Investigación Norteamericanas Association of Research Libraries in North America (ARL) en colaboración con Universidades y otras instituciones académicas. Permite 
el acceso a fuentes de información de ámbito académico.

\section{SPARC Europe}

http://www.sparceurope.org/

Desarrollo complementario de la Scholarly Publishing and Academic Resources Coalition
(SPARC) para el ámbito europeo, y en la que se encuentran colaborando la Universidad de Las Palmas de Gran Canaria y la Universidad Politécnica de Cataluña.

\section{BIBLIOGRAFÍA}

1. Bryce J, Boschi-Pinto C, Shibuya K, Black RE and the WHO Child Health Epidemiology Reference Group. WHO estimates of the causes of death in children. Lancet 2005; 365:1147-1152.

2. Sanz-Valero J, Castiel LD, Wanden-Berghe Lozano C, Juan Quilis V. Internet y la búsqueda de información en Salud Pública: desde la relevancia hacia la "revelancia". Gac Sanit. 2006;20(2):159-60.

3. Jaén Casquero B. Documentación a texto completo. En: Bojo Canales C, Fraga Medín C, Hernández Villegas S, Jaén Casquero MB, Jiménez Planet V, Mohedano Macías L, et al. Internet Visible e Invisible: búsqueda y selección de recursos de información en Ciencias de la Salud. Madrid: Instituto de Salud Carlos III; 2004. p. 65-78.

4. Soros.org [página principal en Internet]. Nueva York (EEUU): Open Society Institute and Soros Foundations Network; [citado 17 jun 2007]. Disponible en: http://www.soros.org/

5. Budapest Open Access Initiative (BOAI) [homepage on the Internet]. Budapest: Open Society Institute; 2002 [citado 17 jun 2007]. Disponible en: http://www.soros.org/openaccess

6. Guerrero R, Piqueras M. Open Acces. A turning point in scientific publication. Int Microbiol. 2004;7:157-161.

7. EPrints.org [homepage on the Internet]. UK: University of Southampton; 2002 [citado 17 jun 2007]. Self Archiving FAQ; [about 37 screens]. Disponible en: http://www.eprints.org/self-faq

8, Veiga de Cabo J. La Biblioteca Virtual en Salud (BVS): una apuesta por la difusión de la producción científica española y latinoamericana en colaboración con la OPS/OMS. Rev Esp Salud Pública. 2001;75(4):277-80.

9. wsis.org [página principal en Internet]. World Summit on the Information Society (WSIS). 